the causal influences between depression and loneliness across three time points (2008, 2012, and 2016) using crosslagged panel (CLP) analysis and examined the trajectories of depression and loneliness in the same time period using latent growth curve (LGC) analysis. Respondents were 2695 adults (aged 51 to 91) from three waves of Health and Retirement Study. The results of CLP model indicated that there was a bidirectional relationship between depression and loneliness, but the effects of loneliness at earlier time points on later depression were larger than that of previous depression on later loneliness. The LGC models showed significant positive associations between the initial levels of depression and loneliness and between the rates of change of these two variables. Significant individual differences were also found in the initial levels and the rates of change for both depression and loneliness. The results further found that age negatively associated with the initial levels of both depression and loneliness but positively related to the rate of change of these two variables. The study highlighted the reciprocal relationship between depression and loneliness and showed the effect of age on the initial levels and rates of change of these two variables.

\section{DEPRESSIVE SYMPTOMS IN THE OLDEST-OLD: THE ROLE OF SENSORY IMPAIRMENTS}

V. Cimarolli1 ${ }^{1}$ D. Jopp ${ }^{2}$, K. Boerner ${ }^{3}$, J. Minahan ${ }^{4}, 1$. Research Institute on Aging, The New Jewish Home, New

York, NY, 2. University of Lausanne, 3. University of Massachusetts Boston, 4. Fordham University, Psychology Department, Bronx, NY, USA

While a fair amount of research has investigated the impact of sensory impairments on the mental health of young older adults (65-79 years of age), only a few studies have focused on the associations of sensory impairments with mental health outcomes in the oldest-old ( 80 years and older). To close this gap, this study examined the separate and combined effects of self-reported vision and hearing impairment for depressive symptoms in a sample of oldest-old individuals, controlling for other mental health risks (e.g., functional disability, health interference, and loneliness). A sample of centenarians and near-centenarians $(N=119$; average age $=99)$ were recruited from the community and geriatric healthcare organizations. In-person interviews were conducted at participants' place of residence. In hierarchical regression analyses vision impairment and its interaction with hearing impairment as well as functional disability, health interference with desired activities, and loneliness were significant predictors of depressive symptoms. Hearing impairment alone was not associated with depressive symptoms, but follow-up analyses clarifying the interaction effect showed that individuals with poor vision had the highest levels of depressive symptoms, if they had a concurrent hearing impairment. Thus, a concurrent presence of poor vision and poor hearing resulted in an increased vulnerability for depressive symptoms. We conclude that given that a majority of oldest-old has sensory impairments which can result in mental health issues, it is important to facilitate this population's access to vision and audiological treatment and rehabilitation.

DEVIATION FROM TYPICAL PATHS AS A MEASURE OF INTRAINDIVIDUAL VARIATION IN LIFESPACE

K. Nielsen ${ }^{1}$, S. Mejia ${ }^{2}$, R. Gonzalez ${ }^{1}$, A. Carmichael ${ }^{1}$, 1. University of Michigan, 2. University of Illinois
Lifespace is typically measured using a self-report questionnaire covering general areas visited over the past 4 weeks (Peel et al., 2005). However, this standard measure does not align with questions about how lifespace ebbs and flows in concert with daily experiences. Thus, methods to quantify perturbations of lifespace at faster timescales are needed. GPS and similar location tracking technologies allow for passive collection of rich data concerning day-to-day travel patterns. While many possible measures can be computed from such location data, we highlight deviation from a typical path as a relevant, dynamic aspect of lifespace across the lifespan. Derivation of this measure involves a two-step procedure. First, we use principal curves to fit one or more paths and estimate the noise in the latitude-longitude paths. Then, we compute the distance of each point from the path. These distance measures can be further summarized to match the research question - for example, a daily average deviation from typical path - and used in additional analyses such as growth curve models. We demonstrate the utility of this method using location data from cell phones of 10 communitydwelling women age 40-65 with moderate to severe bladder incontinence. We anticipate that these women are at risk of lifespace depletion, particularly when their bladder management plan fails. We find that employment, bladder leaks, and trust in their bladder management product all impact estimates of typical daily paths and deviations. This suggests that both inter- and intra-individual patterns in lifespace can be assessed using this technique.

\section{A PROCESS TO INTEGRATE GERONTOLOGICAL KNOWLEDGE INTO RESEARCH ON ETHICAL \& JUSTICE IMPLICATIONS OF POPULATION AGING}

\section{N. Berlinger ${ }^{1}$, K. de Medeiro ${ }^{2}$, 1. The Hastings Center, \\ 2. Department of Sociology and Gerontology, Miami \\ University}

Bioethics is an interdisciplinary field that aims to understand the implications of medical, public health, and biotechnological progress for human identity, relationships, and environments. Bioethics research frames a socially relevant question and seeks a range of perspectives to help answer that question, drawing on empirical methods and on theories of justice informing social policy. Research products may include conceptual frameworks; professional guidance; policy recommendations, and tools for public learning. Bioethics work on topics such as suffering, healthcare decision-making, and resource allocation has long observed that wealthy societies with developed healthcare systems tended to be "aging" societies. Yet to date bioethics research on aging has focused on clinical and end-of-life topics, reflecting the field's origins in considering the implications of life-sustaining medical interventions. In a recent effort to consider the full ethical and social implications of population aging, we raised the research question: What makes a good life in late life? In collaboration with 24 experts, including gerontologists and others who study aging in relation to design, economics, housing, and planning, we completed two workshops to identify core questions, conceptual tools, critical discourses, and data to integrate into bioethics research and support ongoing collaboration with gerontology. Outcomes include a forthcoming special report to be presented via this session, plus a research agenda to widen the bioethics lens from 